\title{
Ultrasensitive refractive index sensor based on twin-core photonic bandgap fibers
}

\author{
Yuan, Scott Wu; Town, Graham E.; Bang, Ole
}

Publication date:

2009

Document Version

Publisher's PDF, also known as Version of record

Link back to DTU Orbit

Citation (APA):

Yuan, S. W., Town, G. E., \& Bang, O. (2009). Ultrasensitive refractive index sensor based on twin-core photonic bandgap fibers. Paper presented at 20th International Conference on Optical Fibre Sensors, Edinburgh, United Kingdom.

\section{General rights}

Copyright and moral rights for the publications made accessible in the public portal are retained by the authors and/or other copyright owners and it is a condition of accessing publications that users recognise and abide by the legal requirements associated with these rights.

- Users may download and print one copy of any publication from the public portal for the purpose of private study or research.

- You may not further distribute the material or use it for any profit-making activity or commercial gain

- You may freely distribute the URL identifying the publication in the public portal

If you believe that this document breaches copyright please contact us providing details, and we will remove access to the work immediately and investigate your claim. 


\title{
Ultrasensitive Refractive Index Sensor Based on Twin-core Photonic Bandgap Fibers
}

\author{
Wu Yuan ${ }^{1, *}$, Graham E. Town ${ }^{2}$, Ole Bang ${ }^{1}$ \\ ${ }^{1}$ DTU Fotonik, Dept. of Photonics Engineering, Technical University of Denmark, \\ DK-2800 Kgs. Lyngby, Denmark \\ ${ }^{2}$ MQ Photonics, Dept. of Physics and Engineering, Macquarie University, NSW 2109, Australia
}

\begin{abstract}
We have theoretically investigated twin-core all-solid photonic bandgap fibers (PBGFs) for evanescent wave sensing of refractive index within one single microfluidic analyte channel centered between the two cores. The sensor can achieve ultrahigh sensitivity by detecting the change in transmission. We find novel features in the sensing characteristics: the sensitivity is higher at the short wavelength edge of a bandgap than at the long wavelength edge, the effective index of the odd supermode $\left(\mathrm{n}_{\text {odd }}\right)$ is more sensitive to ambient refractive index change compared with that of the even supermode $\left(\mathrm{n}_{\text {even }}\right)$.
\end{abstract}

Keywords: fiber sensor, photonic bandgap fiber, directional coupler

\section{INTRODUCTION}

There is a growing research interest in optical fiber refractive index sensors and biosensors using the principle of evanescent wave sensing. Most effort has concentrated on increasing the sensitivity of fiber sensors to detect small refractive index changes. In this context, photonic crystal fibers (PCFs) have received considerable attention recently, as PCF allows infiltration of the analyte into the air holes, thereby maximizing the interaction between the probing electromagnetic field and ambient analyte $[1,2,3]$. Furthermore, due to the flexibility of design and ease of fabrication, multi-core PCF is advantageous for applications in optical communication and sensing $[4,5,6]$. However, fiber sensors based on either conventional single mode fiber (SMF) or PCFs currently cannot compete with some other photonic sensors, e.g., surface plasmon resonance (SPR) sensors. The performance of fiber sensors is currently limited either by the detection limit of around $10^{-5}$ RIU, or by a narrow dynamic range $[1,2,3]$.

PBGFs intrinsically offer higher sensitivity than PCFs for direct refractive index sensing, as in PBGFs a significant part of field of the core modes is present in the high-index rods, whilst in the case of index guiding PCFs, all core modes are expelled from the holes $[4,5]$. Theoretical and experimental studies of multi-core PBGFs have already shown that such fibers have some remarkable coupling properties, e.g., the decoupling and extremes of coupling length $[4,5]$.

In this paper we present the results of modeling of twin-core all-solid PBGFs for evanescent wave sensing of refractive index within one single microfluidic analyte channel centered between the two cores. Our twin-core PBGF operates by simply detecting the change in transmission. Novel features are indentified in their sensing characteristics: the sensitivity is higher at the short wavelength edge of a bandgap than at the long wavelength edge, and the effective index of the odd supermode $\left(\mathrm{n}_{\text {odd }}\right)$ is more sensitive to ambient refractive index change compared with the even supermode $\left(\mathrm{n}_{\text {even }}\right)$.

\section{PRINCIPLE AND MODELING}

Figure 1a shows the modeled all-solid PBGF with two solid cores and one single microfluidic analyte channel $\left(n_{a}\right)$ between the two cores. The cores are separated by two times the pitch $2 \Lambda$ and the cladding is a triangular array of highindex rods of diameter $d=0.53 \Lambda$, where $\Lambda$ is the hole to hole pitch. The fiber is proposed to be fabricated with two different materials, i.e. Poly1 and Poly2. Poly1 will be the lower refractive index background material $\left(n_{b}=1.34\right)$, Poly2 works as the high index $\operatorname{rod}\left(n_{r}=1.53\right)$. Material dispersion has no significant impact on our results and is neglected.

*wyuan@fotonik.dtu.dk; Phone: + (45) 4525 3979, Fax: + (45) 4593 6581; fotonik.dtu.dk 
The twin-core PBGF forms a directional coupler and has broad transmission windows or bandgaps (BGs) delimited by the cut-off wavelengths of the modes of individual high index rods, and were calculated by using MIT plane-wave package [7] as shown in figure $1 \mathrm{~b}$.
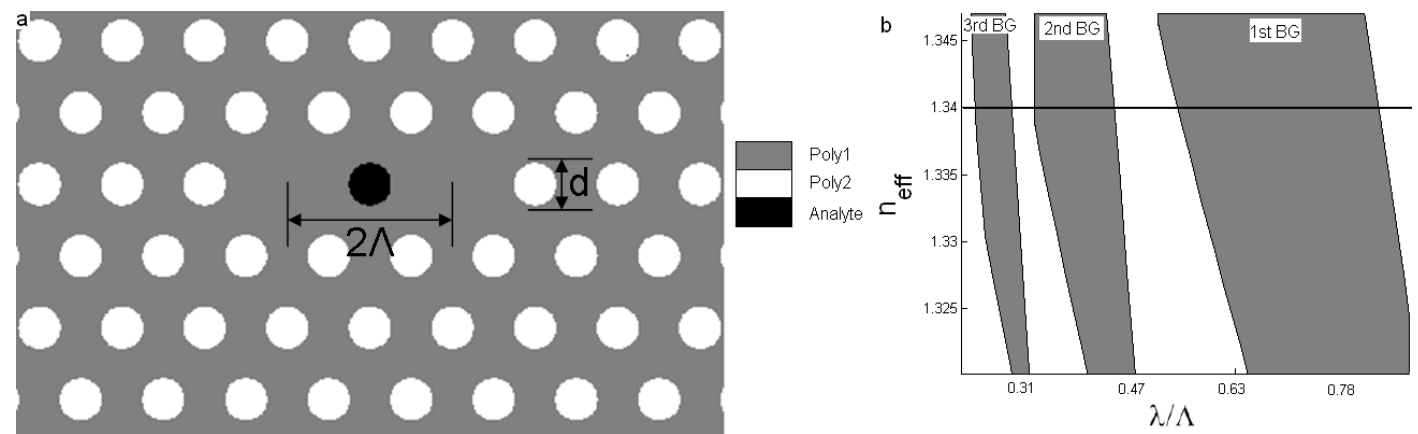

Figure 1. (a) Proposed twin-core PBGF with core separation $2 \Lambda$ and fabricated with Poly1 background and Poly2 rods. (b) Photonic bandgaps of the proposed PGBF plotted with normalized frequency $\lambda / \Lambda$ against the effective index $n_{\text {eff }}$ for indices of refraction $n_{b}=1.34$ and $n_{r}=1.53$. The refractive index of the cores is shown with a solid black line.

In a twin-core PBGF with two identical single-mode cores, the modes of the individual cores interact via their evanescent field and form a pair of supermodes, i.e. symmetric (even) supermode and antisymmetric (odd) supermode. The phase mismatch between the two supermodes will determine the beat length or coupling length $\left(\boldsymbol{L}_{c}\right)$ :

$$
L_{c}=\pi / k\left|n_{\text {odd }}-n_{\text {even }}\right|=\pi / 2 \beta_{c}
$$

Where $\mathrm{n}_{\text {even }}$ and $\mathrm{n}_{\text {odd }}$ are the corresponding effective indices of even and odd supermodes, respectively, $\boldsymbol{k}=2 \pi / \lambda$ and $\lambda$ is the free space wavelength. $\beta_{c}$ is the coupling coefficient between the two cores, which is strongly affected by the confinement of the guided core mode, the separation between the cores, and also the refractive index perturbation of the analytes $\left(\Delta n_{a}\right)$. In other words, $\Delta n_{\text {eff }}=n_{\text {odd }}-n_{\text {even }}=2 \beta_{c} / k$, which depends on the $\lambda, 2 \Lambda$ and $n_{a}$.

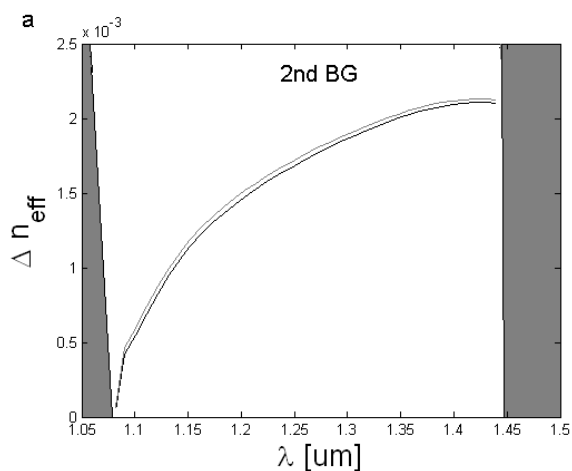

Figure 2. (a) Variation of $\Delta \mathrm{n}_{\text {eff }}$ versus $\lambda$ within $2^{\text {nd }}$ bandgap. (b) Coupling length versus $\lambda$ within the $2^{\text {nd }}$ bandgap, (black solid line: $n_{a}=1.400$, gray solid line: $n_{a}=1.401, \Lambda=3.2 \mathrm{um}$ ). (c) Corresponding coupling length change induced by 0.001 RIU change of the refractive index inside the analyte channel.

In conventional twin-core index-guiding PCFs, $\boldsymbol{L}_{c}$ increases monotonically with frequency and depends primarily on the cores separation [6]. However, in the index-guiding case, because all guided modes are expelled from the holes, a change in the dielectric constant inside the hole cannot induce significant change of $\boldsymbol{L}_{c}$. In contrast, the core-guided modes have a presence in the high-index rods of PBGFs, and the field in the rods plays a key role for the coupling properties of twincore PBGFs. The rod directly between the cores, i.e. the microfluidic analyte channel in our fiber, is particularly important because this is where even and odd supermodes are most clearly different. 
For example, considering the case of the $2^{\text {nd }} \mathrm{BG}$, as shown in figure $2 \mathrm{a}$ and $2 \mathrm{~b}$, when the refractive index of the analyte ( $n_{a}$ ) changes from 1.400 to 1.401 ( $\Delta n_{a}=0.001 \mathrm{RIU}$ ), the $\Delta \mathrm{n}_{\text {eff }}$ curve (black solid line) is shifted upwards (gray solid line) and correspondingly the $\boldsymbol{L}_{c}$ curve (black solid line) is shifted downwards (gray solid line). The key feature in figure $2 \mathrm{a}$ and $2 \mathrm{~b}$ is the steep change of both the $\Delta \mathrm{n}_{\text {eff }}$ and $\boldsymbol{L}_{c}$ curves at the short wavelength edge of the $2^{\text {nd }}$ BG. This indicates that, at short wavelengths, a small refractive index perturbation of the analyte $\left(\Delta n_{a}\right)$ can introduce a significant change to the coupling coefficient $\beta_{c}$ and in turn both the $\Delta n_{\text {eff }}$ and $\boldsymbol{L}_{c}$. This is verified in figure 2c, which shows the change in coupling length $\Delta \boldsymbol{L}_{c}$ induced by a 0.001 RIU change of refractive index inside the analyte channel; a much steeper curve can be achieved at the short wavelength edge than at other parts of the $2^{\text {nd }}$ bandgap.

According to equation (1), if light with wavelength $\lambda$ is incident on one of the cores of a twin-core PBGF, the intensity exiting from the other core will be $I=\sin \left(2 \pi \cdot L \cdot \Delta n_{\text {eff }} / \lambda\right)$, so twin-core PBGFs with constant length $\boldsymbol{L}$ allow for refractive index sensing simply by tracking the variation in transmitted intensity at a fixed $\lambda$. The sensitivity $\partial I / \partial n_{a}$ of this sensing scheme will be:

$$
\partial I / \partial n_{a} \propto(L \cdot 2 \pi / \lambda) \cdot \partial\left(\Delta n_{e f f}\right) / \partial n_{a}
$$

which depends on $\partial\left(\Delta n_{e f f}\right) / \partial n_{a}$ and scales with length $\boldsymbol{L}$. According to the results shown in the inset to figure $2 \mathrm{a}$, $\partial\left(\Delta n_{\text {eff }}\right) / \partial n_{a}$ increases in magnitude as one approaches the short wavelength edge of the $2^{\text {nd }} \mathrm{BG}$.
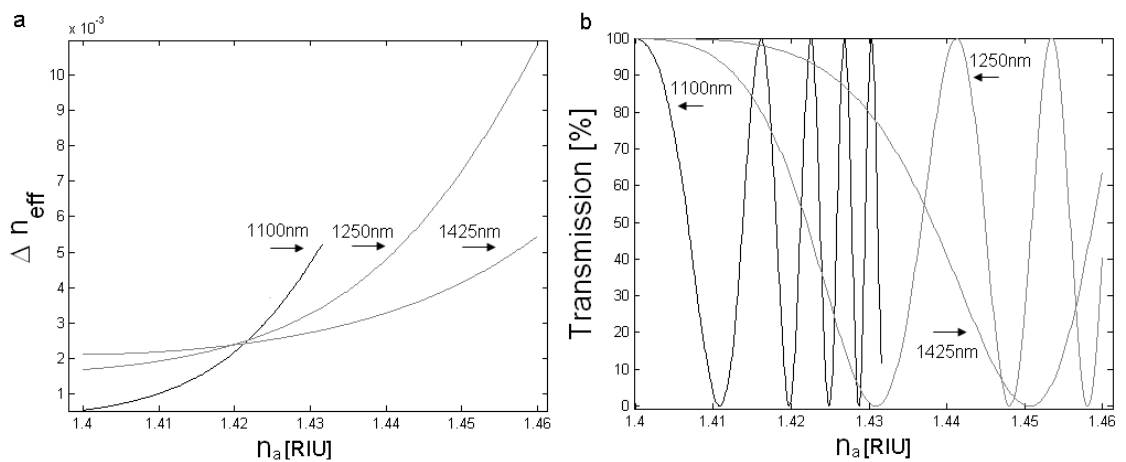

Figure 3. (a) $\Delta n_{e f f}$ of the twin-core PBGF versus $n_{a}$ of analyte at wavelength $1100 \mathrm{~nm}, 1250 \mathrm{~nm}$ and $1425 \mathrm{~nm}$, respectively. (b) The corresponding normalized transmission variation of three specific wavelengths with the change of $n_{a}$. Notes: $\Lambda=3.2 \mathrm{um}$ is applied in all the simulations, the length of fiber is assumed to be the coupling length at each wavelength when the $n_{a}$ is 1.400 .

As demonstrated in figure 3a, the dependences of $\Delta n_{\text {eff }}$ to $n_{a}$ are modeled at three different wavelengths, i.e. $1100 \mathrm{~nm}$, $1250 \mathrm{~nm}, 1425 \mathrm{~nm}$, within the $2^{\text {nd }} \mathrm{BG}$. Among them, the curve for $1100 \mathrm{~nm}$ is much steeper than those of the other two wavelengths. This confirms our previous results that the higher sensitivity can be offered at the short wavelength edge of the $2^{\text {nd }} \mathrm{BG}$. The transmission variations at the three wavelengths are shown in figure $3 \mathrm{~b}$, it is very clear that much more rapid intensity changes are induced by changes in $n_{a}$ at $1100 \mathrm{~nm}$ than at the longer wavelengths. Although the higher sensitivity at $1100 \mathrm{~nm}$ potentially reduces the dynamic range of the sensor, the features of sensitivity and dynamic range can be easily tuned to obtain the desired performance characteristics by an appropriate choice of wavelength.

Interestingly, in the twin-core PBGF, we also find that the variation of $n_{a}$ actually impacts more on the odd supermode than on the even supermode in terms of effective index variation. As shown in figure $4 \mathrm{a}$, the curve of $n_{\text {odd }}$ is much steeper comparing with that of $n_{\text {even }}$ with the variation of $n_{a}$. From the field contours of the even and odd supermodes, shown in the figure $4 \mathrm{~b}$, the analyte channel, which mediates coupling between the core modes, has a significant part of the field localized inside it. The variations of $n_{a}$ from 1.430 to 1.460 strongly impact the field contours of the odd 
supermode whilst leaving the even supermode relatively unchanged. Figure $4 \mathrm{~b}$ also indicates a clear trend of the field of the odd supermode to concentrate in the analyte channel with increasing $n_{a}$. The behaviours of the supermodes with changes in the analyte index between the two guiding cores explain the high sensitivity of our twin-core PBGF. Figure 4a also shows that, at specific $n_{a}$, the ordering of supermodes' effective indices is reversed from $\mathrm{n}_{\text {odd }}>\mathrm{n}_{\text {even }}$ to $\mathrm{n}_{\text {odd }}<\mathrm{n}_{\text {even }}$. It is simply because at this index, the odd supermode reaches the cutoff $n_{b}=1.34 \mathrm{RIU}$, it expands and couples with the higher order modes of the analyte channel.
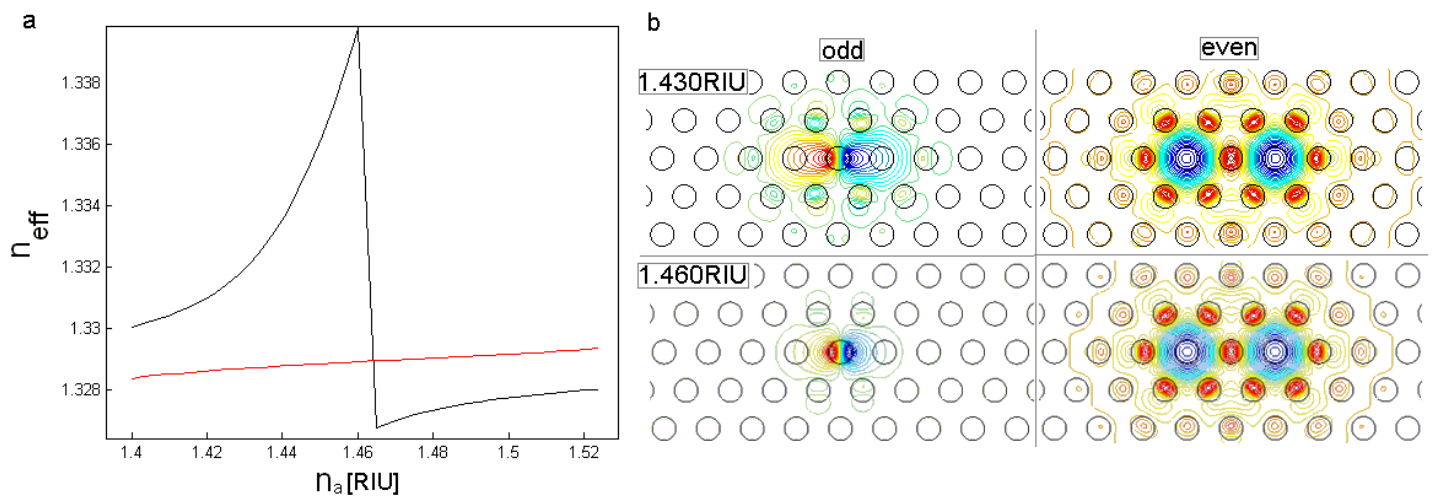

Figure 4. (a) $\mathrm{n}_{\text {odd }}$ of the odd supermode (black solid line) and $\mathrm{n}_{\text {even }}$ of the even supermode (red solid line) $v s n_{a}$ of the analyte at wavelength $1250 \mathrm{~nm}$. (b) Representative field contours of the odd and even supermodes in the twin-core PBGF at $n_{a}=1.430$ and $1.460, \Lambda=3.2 \mathrm{um}$.

\section{CONCLUSIONS}

In this paper, we propose a novel refractive index sensor scheme based on a twin-core all-solid PBGF with one single microfluidic analyte channel centered between the two cores. By detecting the variation in transmission at a fixed wavelength a significantly higher sensitivity can be achieved with this sensor than with twin-core PCFs or twin-core SMF sensors. A typical detection limit magnitude of $10^{-6}$ RIU and dynamic range of 0.01 RIU can be offered by this sensor scheme at wavelength $1100 \mathrm{~nm}$, and the detection limit can be conveniently scaled with length of fiber to over $10^{-7}$ RIU without compromising the dynamic range. Furthermore, the sensitivity and dynamic range of the proposed twincore PBGF strongly depend on the photonic crystal structure, thus appropriate choice of the pitch $(\Lambda)$ and diameter of rod (d) can tailor and further optimize the performance of the proposed refractive index sensor.

\section{REFERENCES}

1. L. Rindorf and O. Bang, "Sensitivity of photonic crystal fiber grating sensors: biosensing, refractive index, strain, and temperature sensing," J. Opt. Soc. Am. B 25, 310-324 (2008).

2. L. Rindorf and O. Bang, "Highly sensitive refractometer with a photonic-crystal-fiber long-period grating," Opt. Lett. 33, 563-565 (2008).

3. D. K. C. Wu, B. T. Kuhlmey, and B. J. Eggleton, "Ultrasensitive photonic crystal fiber refractive index sensor," Opt. Lett.34, 322-324 (2009).

4. Z. Wang, T. Taru, T. A. Birks, J. C. Knight, "Coupling in dual-core photonic bandgap fibers: theory and experiment," Opt. Express 15, 4795-4803 (2007).

5. J. Lægsgaard, "Directional coupling in twin-core photonic bandgap fibers," Opt. Lett. 30, 3281-3283 (2005).

6. J. Lægsgaard, O. Bang, and A. Bjarklev, " Photonic crystal fiber design for broadband directional coupling," Opt. Lett. 29, 2473-2475 (2004).

7. S. G. Johnson and J. D. Joannopoulos, "Block-iterative frequency-domain methods for Maxwell's equations in a planewave basis," Opt. Express 8, 173-190 (2001). 